\title{
Study on Assessment of Preoperative Scoring to Detect Common-Bile-Duct Stones in Patients with Gallstone Pancreatitis
}

\author{
Lloyson Benix A.V. ${ }^{1}$, Mohan L.N. ${ }^{2}$
}

1,2 Department of General Surgery, St. Johns Medical College, Koramangala, Bengaluru, Karnataka, India.

\section{ABSTRACT}

\section{BACKGROUND}

About $44-54 \%$ of acute pancreatitis cases are due to gallstones. $25-30 \%$ of patients have recurrent episodes in another 6 - 18 weeks. The definitive treatment is laparoscopic cholecystectomy at the time of admission. In the case of persistent common bile duct (CBD) stone, if the patient is in cholangitis, endoscopic retrograde cholangiopancreatography (ERCP) is generally recommended. In the absence of cholangitis, performance of elective preoperative ERCP has a low yield. The objectives of this study were to assess quantitative variables for prediction of common bile duct stones in gallstone pancreatitis and to propose a management protocol based on the scoring system.

\section{METHODS}

70 patients based on inclusion and exclusion criteria were selected and their biochemical parameters and radiological reports and intervention results were followed up. A scoring system was proposed based on the parameters and a management protocol was proposed based on the outcome.

\section{RESULTS}

Scores greater than or equal to 3 have a sensitivity of 85 and specificity of 83 to identify retained common bile duct stones in gallstone pancreatitis patients and hence, can be used as a cut off to propose a management protocol.

\section{CONCLUSIONS}

This scoring system can predict the presence of common bile duct stones in non cholangitic biliary pancreatitis patients based on admission laboratory values and initial ultrasonography (USG) findings. A management protocol can be proposed based on the scoring system. It can reduce the number of negative ERCPs and the associated morbidities of ERCP.

\section{KEY WORDS}

Biliary Pancreatitis, Common Bile Duct Stone, ERCP
Corresponding Author: Dr. Lloyson Benix A. V, 1046, Preyara Colony, Mathias Nagar, K.P. Road, Nagercoil, Tamil Nadu, India. E-mail: lloyson@gmail.com

DOI: $10.14260 /$ jemds/2021/17

How to Cite This Article: Benix AVL, Mohan LN. Study on assessment of preoperative scoring to detect commonbile-duct stones in patients with gallstone pancreatitis. J Evolution Med Dent Sci 2021;10(02):83-87, DOI: $10.14260 / \mathrm{jemds} / 2021 / 17$

Submission 20-09-2020,

Peer Review 14-11-2020,

Acceptance 20-11-2020,

Published 11-01-2021.

Copyright (C) 2021 Lloyson Benix A.V. et al. This is an open access article distributed under Creative Commons Attribution License [Attribution 4.0 International (CC BY 4.0)] 


\section{BACKGROUND}

Gallstones are the causative factor of $35-60 \%$ of acute pancreatitis patients in the USA and Western Europe most of which are not severe. But $25 \%$ develop severe complications. ${ }^{1}$ The incidence of acute biliary pancreatitis is higher in women than men and as age increases, the incidence increases about threefold. In about $40 \%$ patients, biliary pancreatitis occurs without any previous warning signs or episodes. Around 4 - 8 percent of cholelithiasis patients eventually develop biliary pancreatitis ${ }^{2}$

Acute pancreatitis is defined by presence of two out of three criteria such as upper abdominal pain, serum amylase or lipase thrice more than the normal limit and the imaging findings.

In 1856, Bernard first proposed the association between gallstones and acute pancreatitis. ${ }^{3}$ This was supported by Opie in 1901 and he published his observations. He noticed in two severe pancreatitis patients that the ampulla of Vater was impacted with gallstones. ${ }^{4}$ Halstead proposed bile reflux into the pancreatic duct led to pancreatitis. ${ }^{5}$ Acosta and colleagues in 1980 proposed that persistent ampullary obstruction due to gallstones worsened pancreatic inflammation. ${ }^{6}$ Neoptolemos suggested a 'multi-hit model' where recurrent ampullary obstruction by gallstones initiated acute pancreatitis. ${ }^{7}$

Stone is a serious risk factor for acute pancreatitis [size less than 2 - $5 \mathrm{~mm}$, multiple stones, irregular surface, mulberry shaped]. ${ }^{8}$ Reflux of bile which is infected with bacteria such as Escherichia coli into the pancreatic duct can be a mechanism which leads to pancreatitis. ${ }^{9}$ Normally, the pressure in the pancreatic duct is thrice than in the common bile duct ${ }^{10}$ and hence prevents bile reflux into pancreatic duct. When ampulla is obstructed, the pressure difference between biliary tree and pancreatic duct may be reversed. ${ }^{11}$

The pathological event is early activation of zymogens inside the pancreatic parenchyma especially activation of trypsinogen to trypsin by enteric peptidase is vital as trypsin activates all other proteolytic precursor enzymes (phospholipase, chymotrypsin, elastase) and other cascades (complement, coagulation and fibrinolysis cascades, kininkallikrein). ${ }^{12}$

The classification proposed by the Atlanta Pancreatitis Classification working group in 2008 most reliably diagnosed acute pancreatitis. ${ }^{13}$ The criteria are acute pain and rebound tenderness in the upper abdomen, increased pancreatic enzyme levels in blood, urine or ascitic fluid thrice more than normal and characteristic radiological findings. Two or more of the above criteria should be fulfilled and other causes of acute abdomen like gastric perforation, acute cholecystitis and an acute myocardial infarction are excluded. It is difficult to differentiate biliary pancreatitis from other forms of acute pancreatitis and needs biochemical and radiological evaluations. The presence of gallstones alone is suggestive of biliary origin although not conclusive. Lipase and amylase concentration in serum rises in $4-8$ hours after an attack of acute pancreatitis and peak after $24 \mathrm{~h}$. They return to normal after $2-4$ days for amylase or after 8 - 14 days for lipase. ${ }^{14}$ Serum lipase has the high sensitivity, specificity and long halflife in serum of about 7 - 13 hours especially in acute alcoholic pancreatitis. ${ }^{15}$ Serum amylase level is usually higher in acute biliary pancreatitis patients than in those with other forms of pancreatitis. ${ }^{16}$ Serum pancreatic enzymes are mostly slightly raised in chronic kidney disease ${ }^{17}$ and serum amylase is high in patients with diseases of the liver, salivary glands, lung or genitalia. ${ }^{18}$

The causative factor of acute pancreatitis must be determined at admission using detailed personal and family history of pancreatic disease, physical examination, laboratory serum tests (i.e., liver enzymes, calcium, triglycerides) and imaging (i.e., right upper quadrant ultrasonography). The personal history should include previous acute pancreatitis, known gallstone disease, alcohol intake, medication intake, known hyperlipidaemia, trauma, recent invasive procedures such as endoscopic retrograde cholangiopancreatography. Abdominal ultrasound is limited by presence of air and fluidfilled bowel loops overlying the pancreas. Computed tomography (CT) is the widely used method to establish and confirm acute pancreatitis. CT findings are classified into pancreatic and peri pancreatic changes. ${ }^{19}$ Pancreatic changes are parenchymal enlargement (diffuse or localized), parenchymal oedema and necrosis. Peripancreatic changes are blurring of fat planes, facial planes thickening and fluid collections. Contrast-enhanced magnetic resonance imaging (MRI) and contrast-enhanced CT have equal diagnostic and prognostic significance in acute pancreatitis. ${ }^{20,21}$ MRI and magnetic resonance cholangiopancreatography (MRCP) are more efficient than CT in detecting bile duct stones, pancreatic haemorrhage, pancreatic duct anatomy, necrotic peripancreatic collections, duodenitis and duodenal narrowing. ${ }^{20-23} \mathrm{MRI}$ is less efficient than CT in detecting small gas bubbles and calcifications. ${ }^{22}$ Endoscopic ultrasound is very sensitive in visualising pancreatic lesions, pseudocysts, common bile duct stones and anatomy of pancreatic duct. ${ }^{24}$

In acute cholangitis patients, urgent ERCP before 24 hours should be done. At present, there is no proven study denoting the optimal ERCP timing for biliary pancreatitis patients without cholangitis. Cholecystectomy during index admission is recommended for mild biliary pancreatitis. In mild biliary pancreatitis planned for interval cholecystectomy, there is an increased risk of readmission due to recurrent biliary events like biliary pancreatitis. In patients with peripancreatic collections, either until the collections resolve or in case they persist, cholecystectomy should be delayed till 6 weeks. In biliary pancreatitis patients who had undergone sphincterotomy and fit for surgery, cholecystectomy should be done because sphincterotomy can prevent recurrent biliary pancreatitis but no other gallbladder induced disease like biliary colic and cholecystitis. ${ }^{25}$ However, ERCP is not indicated in non cholangitic mild biliary pancreatitis; probably not indicated in non cholangitic predicted severe biliary pancreatitis; probably indicated in biliary pancreatitis with common bile duct obstruction; indicated in cholangitic biliary pancreatitis patients. ${ }^{25}$

\section{METHODS}

This was a descriptive study done in St. Johns Medical College and Hospital between 2017 June and 2018 June in a sample size of 70 patients with biliary pancreatitis using convenient sampling technique. The institutional ethics committee approved the study. The inclusion criteria were age more than 18 years with features of biliary pancreatitis like pain and tenderness in the upper abdomen, lipase elevation thrice the 
upper limit of normal (450 U / L) and presence of gallstones in ultrasound at admission. The exclusion criteria included patients who were not willing to participate, patients with cholangitis, patients with severe medical problems that interfered in the performance of ERCP or cholecystectomy and patients with intervention failures. The subject information sheet was given to the patients where the benefits and risks of the study were explained and the confidentiality of the study and the right to withdraw were explained to the patients. Written informed consent was sought from the patients at admission, CBD diameter, amylase, lipase, alkaline phosphatase (ALP), alanine transaminase (ALT ), aspartate aminotransferase (AST), and gamma-glutamyl transpeptidase (GGT), total bilirubin and direct bilirubin were noted. On follow up if liver function test (LFT) is resolved and hepatobiliary scan is normal, laparoscopic cholecystectomy is done. If LFT remains abnormal, then ERCP is done. Based on the finding of the presence or absence of CBD stones, the sample was divided into two groups. Group 1 include patients with CBD stones; Group 2 include patients who did not have CBD stones.

\section{Statistical Analysis}

Chi square and independent sample $t$ tests were done to determine if any significant factors existed between the patient characteristics and laboratory values of the 2 groups. $P$ value of less than or equal to 0.05 was considered as significant. All the significant parameters were assigned a score of 1[26]. The scores and their relation to the two groups (i.e., the presence or absence of CBD stones) were analysed. Their sensitivity and specificity were calculated and an appropriate cut off in the scoring was decided based on the analysis. The management protocol for all the patients was either laparoscopic cholecystectomy or ERCP followed by further procedures. The proportion of positive and negative ERCPS were assessed. So based on the cut off for scoring, management protocol was proposed and was compared with the outcome of ERCPs. All analysis were reviewed with a statistician.

\section{RESULTS}

Of the 70 patients in the study, 14 patients were found to have CBD stones (Group 1) and 56 patients did not have CBD stones (Group 2). Among the patients with CBD stones, 3 were males and 11 were females. And among patients without CBD stones 29 were males and 27 were females. The mean age among the patients in group I is 44 years and mean age among patients in group 2 is 40 years. Upon statistical analysis, the age and gender were not found to be statistically significant ( $p$ less than 0.05).

On analysis it was found the mean CBD diameter of patients in group 1 is $1.01 \mathrm{~cm}$ and of patients in group 2 is 0.76 $\mathrm{cm}$. The $\mathrm{p}$ value is found to be 0.0004 . The mean total bilirubin of patients in group 1 is $3.2 \mathrm{mg} / \mathrm{dl}$ and in group 2 is $2.1 \mathrm{mg} /$ $\mathrm{dl}$. The $\mathrm{p}$ value is found to be 0.008 . The mean direct bilirubin of patients in group 1 is $2.2 \mathrm{mg} / \mathrm{dl}$ and of patients in group 2 is $1.2 \mathrm{mg} / \mathrm{dl}$. The $\mathrm{p}$ value is found to be 0.0038 . The mean ALT of patients in group 1 is $228 \mathrm{IU} / \mathrm{L}$ and of patients in group 2 is $120 \mathrm{IU} / \mathrm{L}$. The $\mathrm{p}$ value is found to be 0.014 . The mean ALP of patients in group 1 is $366 \mathrm{IU} / \mathrm{L}$ and of patients in group 2 is $89 \mathrm{IU} / \mathrm{L}$. The p value is found to be 0.0162 . The mean AST of patients in group 1 is $163 \mathrm{IU} / \mathrm{L}$ and of patients in group 2 is $103 \mathrm{IU} / \mathrm{L}$. The $\mathrm{p}$ value is found to be 0.1332 . The mean GGT in patients of group 1 is $443 \mathrm{IU} / \mathrm{L}$ and in patients in group 2 is $274 \mathrm{IU} / \mathrm{L}$. The $\mathrm{p}$ value is found to be 0.0384 . The mean amylase of patients in group 1 is $2680 \mathrm{IU} / \mathrm{L}$ and of patients in group 2 is $1561 \mathrm{IU} / \mathrm{L}$. The p value is found to be 0.1007 . The mean lipase of patients in group 1 is $5917 \mathrm{IU} / \mathrm{L}$ and of patients in group 2 is $4809 \mathrm{IU} / \mathrm{L}$. The p value is found to be 0.4219 .

So based on the $\mathrm{p}$ values of these various criteria, CBD diameter, ALT, ALP, GGT, total and direct bilirubin were found to be statistically significant. Hence, a scoring was devised based on these criteria giving 1 score for each criterion above a cut-off, i.e., CBD diameter greater than or equal to $9 \mathrm{~mm}$, gamma glutamyl transferase greater than or equal to $350 \mathrm{U} /$ $\mathrm{L}$, alkaline phosphatase greater than or equal to $250 \mathrm{U} / \mathrm{L}$, total bilirubin greater than or equal to $3 \mathrm{mg} / \mathrm{dL}$, direct bilirubin greater than or equal to $2 \mathrm{mg} / \mathrm{dL}$ and ALT greater than or equal to $120 \mathrm{U} / \mathrm{L}$. So scores of 0 - 6 were determined at admission.

\begin{tabular}{|cccc|}
\hline Variable & $\begin{array}{c}\text { Group 1 } \\
\text { With Common Bile } \\
\text { Duct Stone } \\
\text { N =14 }\end{array}$ & $\begin{array}{c}\text { Group 2 } \\
\text { Without Common } \\
\text { Bile Duct Stone } \\
\text { N= 56 }\end{array}$ & P-Value \\
$\begin{array}{c}\text { Common bile } \\
\text { duct size } \\
\text { Total } \\
\text { bilirubin }\end{array}$ & 1.01 & 0.76 & 0.0004 \\
$\begin{array}{c}\text { Direct } \\
\text { Bilirubin }\end{array}$ & 3.2 & 2.1 & 0.0080 \\
ALT & 2.2 & 1.2 & 0.0038 \\
ALP & 228 & 120 & 0.0140 \\
GGT & 366 & 189 & 0.0162 \\
Amylase & 2680 & 274 & 0.0384 \\
Lipase & 5917 & 1561 & 0.1007 \\
AST & 163 & 4809 & 0.4219 \\
\hline \multicolumn{4}{c}{ Table 1. Mean Value of Admission Parameters } \\
\hline
\end{tabular}

On further follow up of the management of these patients, it was found that of the 70 patients, 27 patients had score of zero; 11 patients had score of one; 11 patients had score of two; 8 patients had score of three; 5 patients had score of four; 6 patients had score of five and 2 patients had score of six. Of the 27 patients with score 0 , all 27 belonged to group 2 (i.e., no CBD stones). Of the 11 patients with score 1 , group 2 had 10 patients and group 1 had one patient. Of the 11 patients with score 2 , group 2 had 10 patients and group 1 had one patient. Of the 8 patients with score 3 , group 1 had three patients and group 2 had five patients. Of the 5 patients with score 4 , group 1 had three patients and group 2 had two patients. Of the 6 patients with score 5 , group 1 had four patients and group 2 had two patients. Both the patients with score 2 belonged to group 2 .

\begin{tabular}{|cccc|}
\hline Score & N = 70 & $\begin{array}{c}\text { Group 1 } \\
\text { With Common Bile } \\
\text { Duct Stone N }\end{array}$ & $\begin{array}{c}\text { Group 2 } \\
\text { Without Common- } \\
\text { Bile-Duct Stone N }\end{array}$ \\
0 & 27 & 0 & 27 \\
1 & 11 & 1 & 10 \\
2 & 11 & 1 & 10 \\
3 & 8 & 3 & 5 \\
4 & 5 & 3 & 2 \\
5 & 6 & 4 & 2 \\
6 & 2 & 2 & 0 \\
\hline \multicolumn{4}{c}{ Table 2. Distribution in Group 1 and 2 as per Score } \\
\hline
\end{tabular}


So based on this information, the sensitivity and specificity of this scoring towards predicting CBD stones was analysed. Score 0 had a sensitivity of 100 and specificity of 0 . Score 1 had a sensitivity of 100 and specificity of 48 . Score 2 had a sensitivity of 92 and specificity of 66 . Score 3 had a sensitivity of 85 and specificity of 83 . Score 4 had a sensitivity of 64 and specificity of 82 . Score 5 had a sensitivity of 42 and specificity of 96 . Score 6 had a sensitivity of 0 and specificity of 100 .

\begin{tabular}{|ccc|}
\hline Scores & Sensitivity & Specificity \\
0 & 100 & 0 \\
1 & 100 & 48 \\
2 & 92 & 66 \\
3 & $\mathbf{8 5}$ & $\mathbf{8 3}$ \\
4 & 64 & 92 \\
5 & 42 & 96 \\
6 & 0 & 100 \\
\hline \multicolumn{3}{|c}{ Table 3. Sensitivity and Specificity } \\
\hline
\end{tabular}

The receiver operating characteristic (ROC) curve was plotted for these values and a score of 3 was found to be significant cut off for the above study.

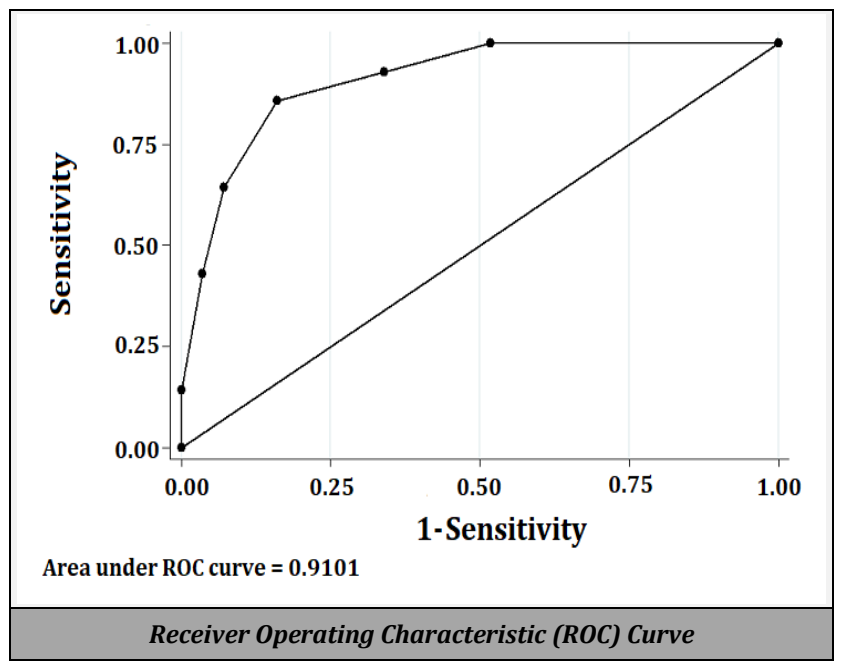

Hence, the values were further analysed for patients with score greater than or equal to 3 and less than 3. 21 patients belonged to the former group of which CBD stones were present in 12 patients and CBD stones were absent in 9 patients. 49 patients belonged to the latter group of which CBD stones were present in 2 patients and CBD stones were absent in 47 patients.

\begin{tabular}{|c|c|c|c|}
\hline CBD Stone & $>=3$ & $<3$ & Total \\
\hline PRESENT & 12 & 2 & 14 \\
\hline ABSENT & 9 & 47 & 56 \\
\hline Total & 21 & 49 & 70 \\
\hline \multicolumn{4}{|c|}{ Table 4. Relation between CBD Stone and Score $>=3$ and Score $<3$} \\
\hline \multicolumn{4}{|c|}{$\begin{array}{l}\text { Sensitivity } 57.1 \text { Specificity } 95.9 \\
\text { Positive predictive value } 85.7 \text { Negative predictive value } 83.9 \\
\text { Accuracy } 84.2\end{array}$} \\
\hline
\end{tabular}

Based on this scoring, a management protocol can be proposed stating patients with score less than 3 can directly go ahead with laparoscopic cholecystectomy and patients with score 3 or more can go ahead with ERCP.

So, in our sample of 70 patients, 32 patients underwent ERCP of whom 14 patients had CBD stones and 18 were negative ERCPs. Based on our scoring, there were 21 patients with score greater or equal to 3 and of which CBD stones were present in 12 patients and CBD stones were absent in 9 patients.

\begin{tabular}{|c|cc|}
\hline Total ERCPs & Sample & $>\mathbf{= 3}$ \\
Positive (with CBD stones) & $\mathbf{3 2}$ & $\mathbf{2 1}$ \\
Negative & 14 & 12 \\
\hline Table 5. Comparison with Respect to ERCPs & \\
\hline
\end{tabular}

This implies only 9 patients must have had negative ERCPs if this management protocol is followed.

\section{DISCUSSION}

Acute pancreatitis is a common complication of gallstones and is the first manifestation in 40 percent of patients who never had any warning signs before. The definitive treatment is laparoscopic cholecystectomy at the time of admission. In case of persistent CBD stone, if the patient is in cholangitis, ERCP is generally recommended. ERCP has its own risk of complications like bleeding, infection, perforation, ascending cholangitis, pancreatitis, cardiopulmonary events and complications of sedation. In the absence of cholangitis, performance of elective preoperative ERCP has generally a low yield. So many studies have been done to predict the presence of CBD stones in biliary pancreatitis patients so as to avoid ERCPs which may end up being negative. Sherman et al. ${ }^{26}$ proposed a scoring based on five parameters CBD diameter, ALP, GGT, total bilirubin and direct bilirubin. His accuracy was $88 \%$. But our study has calculated scoring based on statistically significant variables and we have compared the proposed management protocol with the management done for the patients. We have found that our proposal satisfies our objective which is to reduce negative ERCPs. The percentage of negative ERCPs can be reduced from $56 \%$ to $42 \%$ if the proposed management protocol is followed. Bilal et al. ${ }^{27}$ did a study on choledocholithiasis including patients with pancreatitis, cholangitis, symptomatic cholelithiasis and obstructive jaundice. His scoring was based on the positive predictive value of the scoring. Our study analysed and found a statistically significant cut off of score 3 which changes the line of management. And the proposed management also reduces the negative ERCPs. Our study statistically has found out that if the management was done based on this scoring, the probability of presence of CBD stones is high and hence ERCP becomes a necessity for the patients. Thus, it reduces the chances of negative ERCPs, cost and the complications associated with it. Limitations of this study include the factor that it is a single centre study and if done on a larger scale can improve the sensitivity of the scoring. As we can see, the cut off score 3 was chosen as it had better sensitivity as well as specificity. Also, the availability of endoscopic ultrasound in major tertiary centres may significantly improve the prediction of CBD stones. This was not evaluated in our study and addition of endoscopic ultrasound (EUS) may alter the results of the study. EUS is not readily available in many centres though it is a rapidly developing tool for CBD evaluation. Also, some patients may have lost follow up in our study which becomes a necessity to know if any patients who had underwent laparoscopic cholecystectomy presented postoperatively with CBD stones. 
Also, some patients underwent interval laparoscopic cholecystectomy in view of delayed presentation to the hospital and also because of their personal consent. This may increase the chances of patient developing another episode of pancreatitis or even cholangitis within the next 6 weeks. This may also dilute the study to an extent and doing separate study on index cholecystectomies and interval cholecystectomies will provide further clarity into the study. All these limitations can be worked on and the study can be further improvised to be on a large multicentre basis to further improve the predictive accuracy of the scoring.

\section{CONCLUSIONS}

This scoring system can predict the presence of common bile duct stones in non cholangitic biliary pancreatitis patients based on admission laboratory values and initial USG findings. A management protocol can be proposed based on the scoring system. It can reduce the number of negative ERCPs and the associated morbidities of ERCP.

Data sharing statement provided by the authors is available with the full text of this article at jemds.com.

Financial or other competing interests: None.

Disclosure forms provided by the authors are available with the full text of this article at jemds.com.

\section{REFERENCES}

[1] Frey CF, Zhou H, Harvey DJ, et al. The incidence and casefatality rates of acute biliary, alcoholic and idiopathic pancreatitis in California, 1994-2001. Pancreas 2006;33(4):336-44.

[2] van Erpecum KJ. Gallstone disease. Complications of bileduct stones: acute cholangitis and pancreatitis. Best Pract Res Clin Gastroenterol 2006;20(6):1139-52.

[3] Bernard C. In lecons de physiologie experimentale. Paris: JB Boiliere 1856: p. 278.

[4] Opie EL. The etiology of acute hemorrhagic pancreatitis. Trans Assoc Am Phys 1901;1:314.

[5] Halsted WI. Retrojection of bile into the pancreas: a cause of acute hemorrhagic pancreatitis. Johns Hopkins Hosp Bull 1901.

[6] Acosta JM, Pellegrini CA, Skinner DB. Etiology and pathogenesis of acute biliary pancreatitis. Surgery 1980;88(1):118-25.

[7] Neoptolemos JP. The theory of'persisting'common bile duct stones in severe gallstone pancreatitis. Ann R Coll Surg Engl 1989;71(5):326-31.

[8] Venneman NG, Buskens E, Besselink MGH, et al. Small gallstones are associated with increased risk of acute pancreatitis: potential benefits of prophylactic cholecystectomy? Am J Gastroenterol 2005;100(11):2540-50.

[9] Arendt R, Liebe S, Erdmann K. Biliary pancreatitispathogenesis, therapy, results. Z Gesamte Inn Med 1989;44(13):401-4.
[10] Nitsche R, Fölsch UR. Role of ERCP and endoscopic sphincterotomy in acute pancreatitis. Baillieres Best Pract Res Clin Gastroenterol 1999;13(2):331-43.

[11] Arendt T, Nizze H, Mönig H, et al. Biliary pancreatic refluxinduced acute pancreatitis--myth or possibility? Eur J Gastroenterol Hepatol 1999;11(3):329-35.

[12] Vonlaufen A, Wilson JS, Apte MV. Molecular mechanisms of pancreatitis: current opinion. J Gastroenterol Hepatol 2008;23(9):1339-48.

[13] Banks PA, Bollen TL, Dervenis C, et al. Classification of acute pancreatitis--2012: revision of the Atlanta classification and definitions by international consensus. Gut 2013;62(1):102-11.

[14] Frank B, Gottlieb K. Amylase normal, lipase elevated: is it pancreatitis? A case series and review of the literature. Am J Gastroenterol 1999;94(2):463-9.

[15] Lankisch PG, Burchard-Reckert S, Lehnick D. Underestimation of acute pancreatitis: patients with only a small increase in amylase/lipase levels can also have or develop severe acute pancreatitis. Gut 1999;44(4):542-4.

[16] Dougherty SH, Saltzstein EC, Peacock JB, et al. Rapid resolution of high level hyperamylasemia as a guide to clinical diagnosis and timing of surgical treatment in patients with gallstones. Surg Gynecol Obstet 1988;166(6):491-6.

[17] Montalto G, Lorello D, Carroccio A, et al. Serum trypsin in chronic renal failure and transplant patients. Am J Gastroenterol 1992;87(9):1175-9.

[18] Warshaw AL, Lee KH. Characteristic alterations of serum isoenzymes of amylase in diseases of liver, pancreas, salivary gland, lung and genitalia. J Surg Res 1977;22(4):362-9.

[19] Yeo CJ. Pancreatic pseudocysts, ascites and fistulas. Curr Opin Gen Surg 1994: p. 173-8.

[20] Arvanitakis M, Koustiani G, Gantzarou A, et al. Staging of severity and prognosis of acute pancreatitis by computed tomography and magnetic resonance imaging-a comparative study. Dig Liver Dis 2007;39(5):473-82.

[21] Štimac D, Zrnic IK, Radic M, et al. Outcome of the biliary acute pancreatitis is not associated with body mass index. Pancreas 2007;34(1):165-6.

[22] Piironen A. Severe acute pancreatitis: contrast-enhanced CT and MRI features. Abdom Imaging 2001;26(3):225-33.

[23] Ward J, Chalmers AG, Guthrie AJ, et al. T2-weighted and dynamic enhanced MRI in acute pancreatitis: comparison with contrast enhanced CT. Clin Radiol 1997;52(2):10914.

[24] Rizk MK, Gerke H. Utility of endoscopic ultrasound in pancreatitis: a review. World J Gastroenterol 2007;13(47):6321-6.

[25] Johnstone C. Pathophysiology and nursing management of acute pancreatitis. Nurs Stand 2018;33(4):75.

[26] Sherman JL, Shi EW, Ranasinghe NE, et al. Validation and improvement of a proposed scoring system to detect retained common bile duct stones in gallstone pancreatitis. Surgery 2015;157(6):1073-9.

[27] Al-Jiffry BO, Khayat S, Abdeen E, et al. A scoring system for the prediction of choledocholithiasis: a prospective cohort study. Ann Saudi Med 2016;36(1):57-63. 